\title{
Capital Social y Pobreza en Ecuador, 2010-2012
}

\section{Social Capital and Poverty in Ecuador, 2010-2012}

\section{Capital Social e Pobreza em Equador, 2010-2012}

\section{Héctor Alberto Botello Peñaloza** - Colombia}

Recibido el 23 de septiembre de 2013 - Aprobado el 6 de noviembre de 2013

Este trabajo muestra resultados parciales del proyecto de investigación "Capital Social y su influencia en el desarrollo económico del Ecuador”, programado y financiado por la Universidad Industrial de Santander, UIS.

** Economista Universidad Industrial de Santander. Estudiante de Maestría en Ingeniería Industrial de la Universidad Industrial de Santander. Colombia. Correo: hectoralbertobotello@gmail.com 


\title{
Resumen
}

Objetivos: evidenciar, estadísticamente, cómo el Capital Social contribuye a la reducción de la probabilidad de que un hogar sea pobre en Ecuador, entre 2010 y 2012. Las redes sociales que crean las personas entre sí, son consideradas como uno de los factores que permiten minimizar la exposición de los hogares a la pobreza. Este fenómeno de cohesión se denomina capital social. Metodología: enfoque cuantitativo, se estimó un modelo probabilístico para encontrar la relación que existe entre ser pobre y la participación de los hogares en las redes sociales. El análisis se hizo a través de los datos de las encuestas de hogares entre 2010 y 2012 en Ecuador del módulo de participación social. Resultados: no se encontró evidencia estadística para sustentar que el Capital Social es una variable que contribuya a la reducción de pobreza. No obstante, sí se evidenció una reducción continua en la probabilidad de los hogares ecuatorianos de ser pobres, controlando una gran variedad de factores socioeconómicos, como la educación del jefe de hogar, la composición del hogar, la ubicación y la edad del jefe de hogar, entre otros. Conclusiones: la cohesión social no es un factor por el que los hogares de Ecuador puedan salir de la pobreza, lo cual puede ser negativo en la aplicación de programas sociales que vinculen a la comunidad.

Palabras claves: Capital Social, Participación comunitaria, Pobreza monetaria, modelos probit, logit.

\begin{abstract}
Objective: To statistically demonstrate how Social Capital contributes to reducing the probability of a household being poor in Ecuador, between 2010 and 2012. Social networks are considered one of the factors that minimize the exposure of households to poverty. This cohesion phenomenon is called Social Capital. Methodology: the study has a quantitative approach. A probabilistic model was used to determine the relationship between being poor and the participation of household in social networks. Data was analyzed from the section about social participation in household surveys conducted between 2010 and 2012 in Ecuador. Results: No statistical evidence was found that supported the theory that Social Capital is a variable that contributes to reduce poverty. Nevertheless, the continuous reduction in the probability of Ecuadorian households being poor was evident, controlled by a wide variety of socio-economic variables, such as the education of the head of a household, household structure, and the location and age of the head of the household, amongst others. Conclusions: Social cohesion is not a factor by which households from Ecuador can overcome poverty, which can be a negative factor in the implementation of social programs involving the community.
\end{abstract}

94 git model.

Keywords: Social Capital, Community Participation, Monetary poverty, Probit model, Lo- 


\section{Resumo}

Objetivos: evidenciar, estatisticamente, como o Capital Social contribui à redução da probabilidade de que um lar seja pobre em Equador, entre 2010 e 2012. As redes sociais que criam as pessoas entre sim, são consideradas como um dos fatores que permitem minimizar a exposição dos lares a pobreza. Este fenómeno de cohesión se denomina capital social. Metodologia: enfoque quantitativo, estimou se um modelo probabilístico para encontrar a relação que existe entre ser pobre e a participação dos lares nas redes sociais. O analises se fez a través dos dados das enquetes de lares entre 2010 e 2012 em Equador do módulo de participação social. Resultados: não se encontrou evidencia estatística para sustentar que o Capital Social é uma variável que contribuía à redução de pobreza. Não obstante, se evidenciou uma redução continua na probabilidade dos lares equatorianos de ser pobres, controlando uma grão variedade de fatores socioeconômicos, como a educação de chefe de lar, a composição do lar, a ubiquação e a idade do chefe de lar, entre outros. Conclusões: a coesão social não é um fator pelo que os lares de Equador possam sair da pobreza, o qual pode ser negativo na aplicação de programas sociais que vinculem à comunidade.

Palavras chave: Capital Social, Participação comunitária, Pobreza monetária, modelos probit, logit. 


\section{Introducción}

Según el Informe Panorama Social 2009-2010, presentado por la Comisión Económica para América Latina y el Caribe (CEPAL, 2010), la crisis económica internacional provocó un aumento de 180 a 184 millones de pobres en América Latina durante el 2008 y 2009. Igualmente, las personas en situación de indigencia aumentaron de 71 a 73 millones, equivalente al 13\% de la población regional. Según el mismo informe, la pobreza en los niños es 1,7 veces más alta en menores de 15 años que en adultos, y 1,15 veces mayor en mujeres que en hombres. El informe también concluye que los efectos de la crisis internacional pueden retrasar los logros obtenidos en términos de eliminación de la pobreza en la última década.

Para evitar estos efectos sobre la pobreza, es necesario identificar los determinantes de esta condición y las formas de protección que tienen los hogares en contra del aumento de la misma; factores que influirán en el diseño de las políticas del gobierno para hacer frente a este fenómeno.

En este sentido, los individuos pueden emplear diferentes estrategias para afrontar de mejor manera las fluctuaciones de la actividad económica y la reducción de sus ingresos. Una de ellas, es el apoyo y la cohesión social que tienen los individuos de las comunidades donde habitan, característica conocida en la literatura científica como Capital Social. Es por esto que el este trabajo busca encontrar evidencia empírica que vincule el capital social y la pobreza en en Ecuador, para examinar si los hogares que poseen una dotación de capital social más alto reducen sus probabilidades de ser pobre.

A continuación, se repasan los diferentes conceptos con la intención de explorar la naturaleza del término y las diferentes formas que se han planteado para medirlo.

\section{El Capital Social}

El concepto parte de una noción de origen sociológico (Putnam, 2001), dado que fueron las ciencias sociales, las primeras en analizar algunos de sus elementos constitutivos, tales como la solidaridad, la confianza, la reciprocidad, las redes sociales, etc. Se entiende, entonces, que el capital que el capital social tiene una visión de estudio interdisciplinaria (Robinson, Siles y Schmid, 2002). Sin embargo, la definición de capital social ha sido abordada desde diferentes puntos de vista a través de los años (Woolcock y Narayan, 2000). 
Putnam (2001) ${ }^{1}$ define Capital Social como la pertenencia a asociaciones civiles y sus características asociadas, como las normas implícitas y la confianza entre los participantes; esta dinámica favorece la acción y la cooperación para beneficio mutuo. Estos fenómenos acrecientan los beneficios de la inversión en capital físico y humano, ya que reduce los costes de la información para las transacciones y los desenvolvimientos en el mercado laboral. Con esta definición, Putnam pone el acento en el componente comunitario del Capital Social y no simplemente como un recurso individual (Putnam, 1993).

Desde otro punto de vista, Bourdieu (1986) define el Capital Social como la sumatoria de recursos tangibles e intangibles que acumulan los individuos o los grupos en función de redes, más o menos institucionalizadas de conocimientos y reconocimiento mutuo. Coleman (1988) complementó este enfoque, afirmando que el Capital Social no es una entidad simple de definir, sino una variedad de diferentes entidades, con dos elementos en común: su existencia facilita ciertas acciones de los actores y todos ellos consisten en algún aspecto de la estructura social.

Un concepto que impulsó un análisis más detallado se relaciona con la conformación de las redes sociales. Este fue introducido por Putnam (1993), para referirse al Capital Social como las conexiones entre individuos, donde se generan normas y confianza que permiten a los participantes actuar de manera más eficaz en función de objetivos comunes.

Esta definición ha influenciado a otras disciplinas. Algunos elementos que se desprenden, incluyen el enfoque en las relaciones interpersonales y sus efectos sobre la eficiencia del intercambio social en la provisión de bienes o la mejor organización del mercado (Granovetter; 1973, 1985; Chantarat y Barrett 2012). Sobre esta línea es la que se apoya el presente trabajo; es decir, sobre el efecto económico sobre la pobreza que puede tener la utilización del Capital Social en la economía; sobre el particular se han definido diferentes esquemas presentados a continuación.

\section{Capital Social en la economía}

Las teorías ortodoxas del desarrollo económico se basan sobre la idea tradicional de crecimiento económico, en la cual los factores que determinan la tendencia de crecimiento, a largo plazo de las economías, son el capital físico

1 En una revisión de la literatura existente, Putnam reconoce la aparición del término de capital social en 1977. Según Putnam (2000) este término se le atribuye a Lyda Judson Hanifan precursora en la utilización del mismo, en un estudio sobre educación rural y el papel de una comunidad involucrada en el éxito de las escuelas. 
(Solow, 1957), y la presencia de una fuerza de trabajo calificada (Romer, 1990). Sin embargo, autores en el desarrollo y el crecimiento resaltan la importancia de la cohesión social y el desarrollo sostenible para que las sociedades prosperen (Knack y Keefer 1997; Putnam 1993; Narayan 1997)

Granovetter (1995) afirma que casi todo el comportamiento económico está incrustado en las redes de relaciones sociales. El Capital Social facilita a los agentes del mercado la realización de las transacciones, gracias a la confianza y a un mayor acceso a la información (Saracostti, 2010), lo que facilita la coordinación de intercambios, y la reducción del comportamiento irracional, a través de transacciones repetitivas (Dasgupta, 2000). En este orden de ideas, el Capital Social desempeña un papel importante en la conformación de los resultados de la acción económica, en lo micro y macroeconómico (Rodrik, 1997). Algunos autores (Cleaver, (2005); Collier (2002), hacen énfasis en la utilización del Capital Social como instrumento para la promoción del crecimiento económico y la reducción de la pobreza en forma sostenible; las teorías que describen estas interrelaciones se explican a continuación.

\section{El Capital Social y su vínculo con la pobreza}

La teoría del Capital Social sugiere que a medida que aumenta el Capital Social dentro de las redes de partícipes en las transacciones, los costes de transacción se reducen y con la puesta en marcha de normas la disparidad de beneficios se reduce y se eleva el nivel medio de beneficios. Esta relación inversa entre la disparidad de ingresos y el promedio de ellos, es en parte el resultado de los términos de intercambio en las redes ricas en Capital Social, que favorecen a los menos privilegiados y reducen la disparidad gracias a un mayor volumen de inversiones en bienes públicos, ofrecido con independencia del ingreso individual. Junto con el uso de las reglas sociales que aplican a quienes comparten el Capital Social, lo que les permite aspirar a una mayor igualdad de oportunidades.

Para Collier (2002) el Capital Social puede ser utilizado entre las personas pobres para cubrirse contra el riesgo o choques externos, tal como las enfermedades o los daños causados por las inclemencias del clima. En este sentido, se puede recurrir a la puesta en común de sus recursos, como el cuidado de los alimentos, préstamos temporales o el cuidado de los niños (Lahn, 2012; Schneider, 2005; Caughy y O’Campo, 2006). Asimismo, las redes sociales facilitan la puesta en marcha de micro negocios cooperativos que pueden contribuir al aumento

98 temporal de los ingresos (Das, 2004).

Para citar este artículo: 
En el aspecto comunitario Grootaert (2001) y Narayan $(1997,1999)$ hacen énfasis en el Capital Social como proveedor de bienes y servicios que el mercado o el gobierno no pueden proveer directamente. Por ejemplo, la función del Capital Social en las comunidades como generador y transmisor de la información; como lo exponen Narayan y Pritchett (1999) al mencionar el caso de Tanzania, donde las pequeñas comunidades se reúnen y dialogan sobre métodos agrícolas alternativos o sobre la existencia de recursos naturales que son escasos como las fuentes de agua y madera:

1. Hassan y Birungi (2011) resaltan como el Capital Social influye sobre la pobreza:

2. Promueve la transmisión de los conocimientos acerca de la tecnología y los mercados, reduciendo la incertidumbre y los costos de transacción (costos de obtener información acerca de la tecnología, el mercado, la calidad crediticia de las partes del contrato, entre otros) reduciendo la pobreza.

3. Gracias a la interacción social, se pueden reducir los problemas de free rider.

4. La vigilancia de la prestación de servicios y bienes públicos eficaces.

5. Mejorar el acceso a los mercados o las limitaciones de crédito y, por tanto, reducir la vulnerabilidad de los hogares a la pobreza.

Es necesario resaltar que no son pocos los trabajos empíricos que vinculan al Capital Social con pobreza; en especial los trabajos impulsados en de los países en desarrollo. Por ejemplo, los estudios sobre la influencia que tienen las asociaciones agrícolas sobre la pobreza monetaria en Uganda, fueron llevados a cabo por Hassan y Birungi (2011). Estos autores investigan cómo el Capital Social puede tener un impacto en la pobreza del hogar, medida por el ingreso del hogar. El Capital Social se midió como el porcentaje de personas que pertenecen a una variedad de organizaciones sociales; un acercamiento teórico al dado por Putnam (1993). Esta variable se introduce en una ecuación determinística junto con otras variables explicativas como la educación, el sexo del jefe de hogar, edad, número de personas en el hogar etc. Entre los resultados los autores observaron que el gasto de los hogares se asocia positiva y significativamente con la formación de Capital Social y la participación del grupo. Ellos estiman que el impacto del Capital Social sobre el bienestar del hogar se compara bien con el de otras formas de acumulación de capital, como el del capital humano. Pero al mismo tiempo, este tipo de capital ejerce 
una fuerte influencia positiva en la probabilidad de participar en grupos sociales. El trabajo finaliza con recomendaciones sobre la implementación de programas públicos que fortalezcan las instituciones sociales entre las comunidades para así fomentar el crecimiento y reducir la pobreza.

Similares trabajos existen para indonesia y Bolivia. En el caso del primero Grooteart (1999) prueba la causalidad de dos vías entre el bienestar del hogar y el stock de Capital Social acumulado por los hogares en algunas comunas (villas) de Indonesia. La estimación la realiza por medio de una función del ingreso del hogar contra un conjunto de variables que incluyen variables asociadas al Capital Social que se aproxima mediante la intervención en asociaciones comunitarias. Para el cálculo utilizan un test de medias y regresión lineal. Para calcular el Capital Social en las comunidades, el autor calcula un indicador sintético donde incluye seis dimensiones, entre ellas: la diversidad racial presente, la densidad de población dentro de la misma, y el número de asociaciones dentro de la comunidad. Con todas las variables introducidas se obtienen los siguientes resultados:

- El Capital Social muestra una relación positiva con el gasto del hogar $(+3,4 \%)$. El autor apunta que el efecto de esta variable es similar al que tiene el capital humano del jefe de hogar.

- El aumento de un 20\% en el número de personas dentro de la asociación incrementa el gasto de los hogares per cápita en un $1.5 \%$.

- El aumento de un 20\% de la participación dentro de las asociaciones incrementa el gasto en un $3.2 \%$.

- El Capital Social genera externalidades positivas como la mayor cantidad de activos tangibles e intangibles dentro del hogar, mayor capacidad de ahorro y un mejor acceso al crédito. Estos aspectos le confieren características de bien público, que se debe apoyar en políticas públicas para su promoción y expansión.

En el segundo trabajo, Grooteart y Narayan, (2004) llegan a la conclusión de que una mayor participación de los hogares en asociaciones comunitarias disminuye la probabilidad de ser pobre, en Bolivia. En esta investigación el Capital Social se midió en seis dimensiones, metodológicamente similares a las de Grooteart (1999).

Entre los resultados se obtuvo que los retornos del Capital Social son 2.5 más altos que la educación. Un incremento del Capital Social en un 25\% incrementa el gasto del hogar en un 10.5\% mientras que el incremento similar en la dotación

100 del capital humano dentro del hogar, incrementa el gasto en $4.5 \%$. 
Asimismo, los hogares con bajos ingresos y poca tenencia de tierras son los que tienden a hacer más inversiones en este factor. $\mathrm{Y}$ al igual que en el estudio de Indonesia, la dotación de Capital Social generan otras externalidades como un mayor acceso al crédito y a servicios públicos.

En complemento a los trabajos anteriores, el presente estudio busca encontrar evidencia de cómo el Capital Social influencia la pobreza de los hogares en Ecuador, en el periodo de 2010 a 2012.

\section{Metodología}

A continuación se presenta la metodología implementada en el trabajo. Primero, se expone la forma cómo se cuantificó el Capital Social, después se indica el modelo que muestra la forma de medirlo y al final se evidencian los resultados de las estimaciones en los hogares ecuatorianos.

\section{Cuantificando el Capital Social}

Existe un debate permanente en la delimitación del Capital Social en los trabajos empíricos (Putnam, 2001). El enfoque que se presenta en este trabajo es el que mide el Capital Social en términos de la pertenencia a organizaciones locales (Hassan y Birungi, 2011). Esta idea ha sido aplicada en numerosos estudios de Capital Social (Putnam, 1993; Grooteart, 1999; Hassan y Birungi, 2011) gracias a que es fácil de introducir y de implementar en modelos estadísticos de comparación.

Se utiliza la base de datos de empleo de Ecuador publicada por el Instituto Nacional de Estadística (INEC)² de este país, entre los años de 2010 y 2012. Dentro de la base de datos existen preguntas que permiten caracterizar el concepto de Capital Social escogido para este trabajo, aproximación a la visión de Putnam (1993) sobre la participación de los individuos en la comunidad a través de asociaciones comunitarias ${ }^{3}$.

Específicamente, en la base de datos se pregunta a las personas del hogar si han participado en actividades comunitarias. Estas incluyen las mingas ${ }^{4}$, cons-

2 Para consultar la naturaleza metodológica de la encuesta, se puede revisar en el siguiente link http:// www.inec.gob.ec/estadisticas/index.php?option $=$ com_remositoryyItemid $=y$ func $=$ downloadyid $=17$ 6ychk=2f0f6e72d1dd5f1ba5e3acb573f5780byno_html=1ylang=es

3 Enfoque cuantitativo escogido por otros trabajos tales como Restrepo, (1998), Arriagada (2005) y Londoño (1997).

4 Según Castillo (1989) la minga es una forma comunitaria de asociación donde las personas pertenecientes, realizan actividades no remuneradas en beneficio común. 
trucciones comunales, reuniones comunitarias, asociaciones civiles y cooperativas. Estas respuestas son configuradas de tal forma que permiten la construcción de una variable binomial que marca la pertenencia o no a una asociación comunitaria, variable que se escogió como proxy de la tenencia de Capital So$\mathrm{cial}^{5}$. La evolución de esta variable se muestra en la Figura 1. En términos de distribución por tipo de organizaciones, se encontró que el 4,21\% de los hogares ecuatorianos participan en mingas, le sigue las reuniones comunitarias con el $2,72 \%$ y la intervención en la infraestructura comunitaria.

En la gráfica 1 se aprecia que la tendencia en el nivel de participación de los hogares ecuatorianos en las asociaciones comunales es decreciente. Desde el 19\% de los hogares en 2010 a 8,95\% en el 2012. Por tipo de organización, la participación en migas ha sido la de mayor reducción pasando del $9.5 \%$ de los hogares al $4.5 \%$. Seguidas por las reuniones de la comunidad, que registraron el 8,65\% de los hogares en 2010 y solo el 3\% de los hogares en 2012. Por otro lado, las instancias civiles y las cooperativas han incrementado marginalmente el número de hogares que participaron en estas desde el $0,21 \%$ al $0,31 \%$ y del $0,32 \%$ al $0,39 \%$, respectivamente.

5 En este trabajo no se toma en cuenta la intensidad con la cual las personas participan de estas asociaciones. No obstante se consideraría importante analizar en el futuro cuál es el efecto del número de horas que los hogares participan en las asociaciones comunitarias y su influencia en la tenencia de capital social, ya que un mayor número de horas mostraría niveles más elevados de capital social. 


\section{Gráfica 1. Porcentaje de Hogares que participan en actividades comunitarias por tipo de asociación. Años 2010 - 2012.}

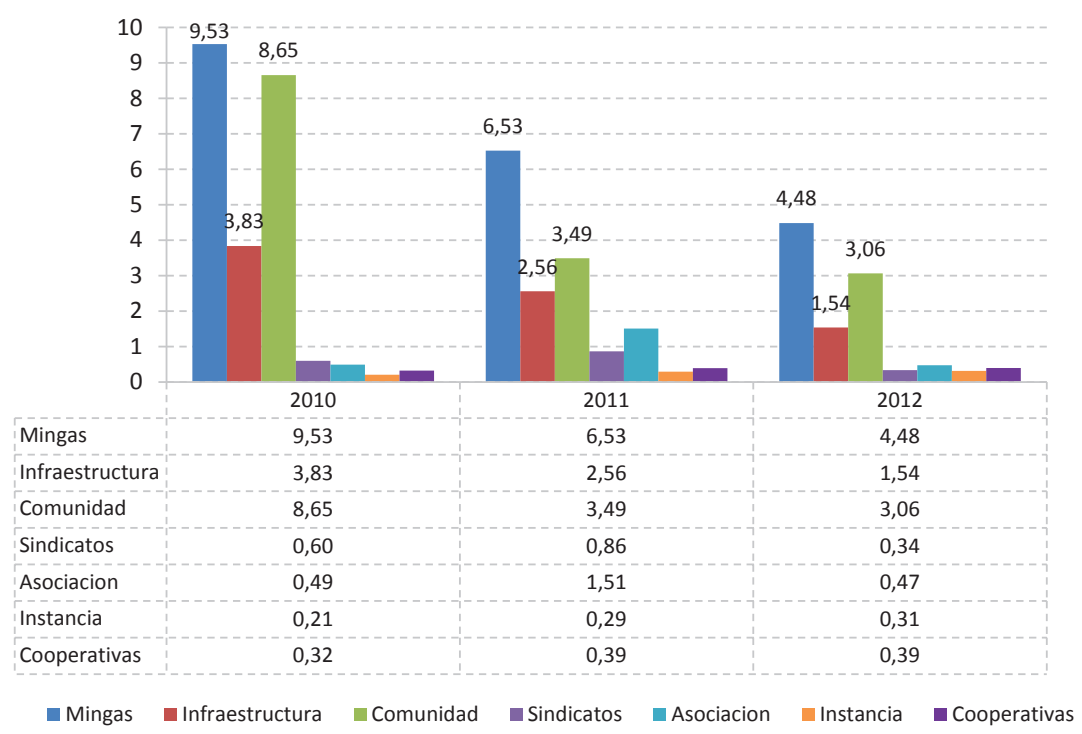

Fuente: Banco Central de Ecuador. Instituto Nacional de Estadística con base en la Encuesta de Empleo, Subempleo y Desempleo 2010-2012.

Mapa 2. Porcentaje de Hogares que participan en asociaciones comunitarias en por provincias en Ecuador Años 2010 y 2012.

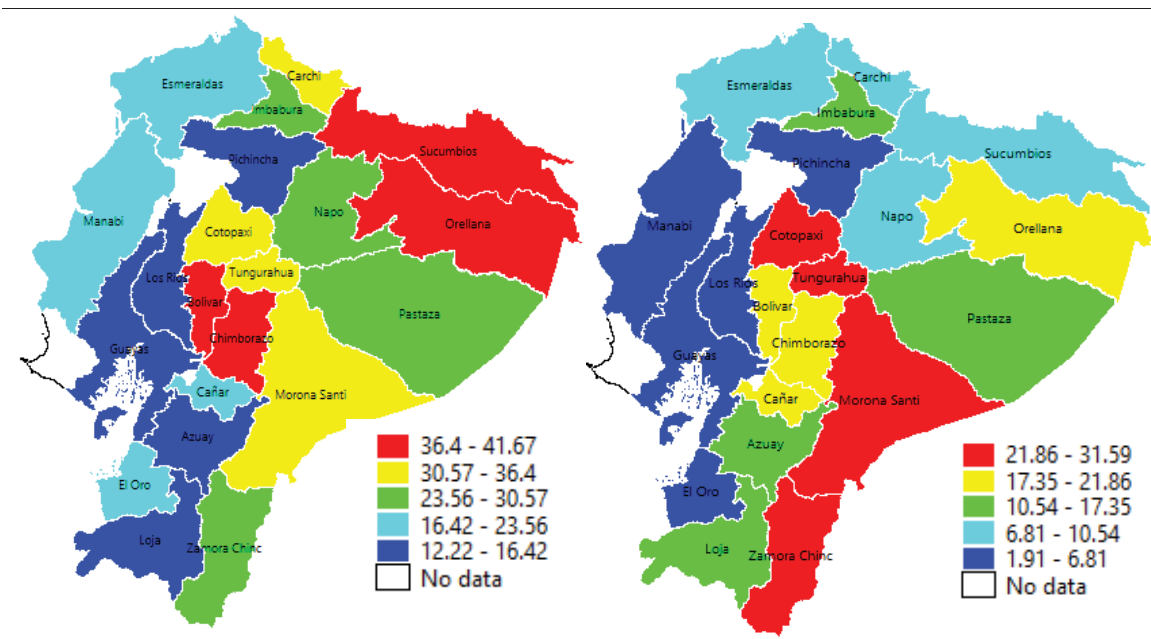

Fuente: Cálculos del autor con base en los datos del Banco Central de Ecuador, el Instituto Nacional de Estadística y la Encuesta de Empleo, Subempleo y Desempleo 2010-2012. 
Tabla 1. Porcentaje de Hogares que participan en actividades comunitarias en Ecuador por características seleccionadas del jefe de hogar. Años 2010-2012

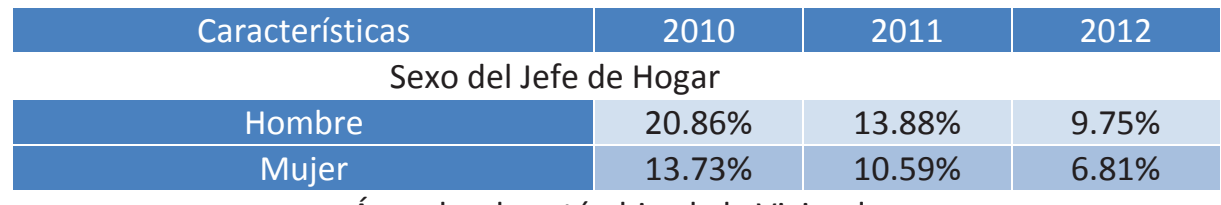

Área donde está ubicada la Vivienda

\begin{tabular}{|c|c|c|c|}
\hline Urbana & $11.90 \%$ & $7.37 \%$ & $3.84 \%$ \\
\hline Rural & $33.97 \%$ & $24.16 \%$ & $19.15 \%$ \\
\hline \multicolumn{1}{|c|}{ Raza del Jefe de Hogar } \\
\hline Indígena & $53.81 \%$ & $33.63 \%$ & $33.03 \%$ \\
\hline Afroecuatoriano & $21.33 \%$ & $17.04 \%$ & $10.94 \%$ \\
\hline Negro & $26.63 \%$ & $14.85 \%$ & $6.86 \%$ \\
\hline Mulato & $12.68 \%$ & $10.82 \%$ & $8.78 \%$ \\
\hline Montubio & $19.50 \%$ & $10.00 \%$ & $9.66 \%$ \\
\hline Mestizo & $16.42 \%$ & $11.43 \%$ & $7.22 \%$ \\
\hline
\end{tabular}

Nivel Educativo del Jefe de Hogar

\begin{tabular}{|c|c|c|c|}
\hline Ninguno & $20.86 \%$ & $13.88 \%$ & $9.75 \%$ \\
\hline Centro de alfabetización & $13.73 \%$ & $10.59 \%$ & $6.81 \%$ \\
\hline Primaria & $26.33 \%$ & $16.36 \%$ & $14.97 \%$ \\
\hline Educación Básica & $37.53 \%$ & $35.77 \%$ & $15.95 \%$ \\
\hline Secundaria & $23.13 \%$ & $15.84 \%$ & $11.38 \%$ \\
\hline Superior no universitaria & $17.95 \%$ & $23.16 \%$ & $8.37 \%$ \\
\hline Universitaria & $14.26 \%$ & $9.66 \%$ & $5.57 \%$ \\
\hline Post-grado & $12.17 \%$ & $13.49 \%$ & $7.85 \%$ \\
\hline
\end{tabular}

Quintiles de Ingreso

\begin{tabular}{|c|c|c|c|}
\hline 1 & $18.51 \%$ & $12.18 \%$ & $9.69 \%$ \\
\hline 2 & $22.87 \%$ & $15.26 \%$ & $12.30 \%$ \\
\hline 3 & $20.38 \%$ & $13.29 \%$ & $8.62 \%$ \\
\hline 4 & $17.14 \%$ & $13.08 \%$ & $7.80 \%$ \\
\hline 5 & $14.60 \%$ & $11.19 \%$ & $6.90 \%$ \\
\hline
\end{tabular}

Fuente: Cálculos del autor con base en los datos del Banco Central de Ecuador, el Instituto Nacional de Estadística y la Encuesta de Empleo, Subempleo y Desempleo 2010-2012.

En relación con las características del hogar, se puede concluir que los hogares rurales con jefes de hogar hombres, de etnia indígena, con pocos ingresos económicos y sin ningún nivel educativo son más propensos a participar en 104 actividades comunitarias. 
Por disposición geográfica, se observa en el mapa 1 las variaciones de participación de los hogares en actividades comunitarias entre el 2010 y el 2012. En términos generales, en las regiones de Morona Santiago $(31,59)$, Cotopaxi $(23,79)$, Tungurahua $(23,42)$, Zamora Chinchipe $(22,4)$, Orellana $(21,86)$ se encuentran los mayores porcentajes de hogares con participación en sus comunidades.

Sobre la variación en el periodo estudiado, se aprecia una baja generalizada en todas las regiones. Esta fue significativamente mayor en las provincias de Chimborazo (-23,42), Carchi $(-25,97)$, Sucumbíos $(-30,9)$; por tipo, la organización que más influye fue la minga. Mientras que en las provincias de Azuay $(-0,9)$, Loja $(-2,1)$ y Morona Santiago $(-4,11)$ la variación fue mínima.

\section{Modelo}

Para realizar las estimaciones se aplicó un modelo probabilístico, que relacione la probabilidad de ser pobre frente al Capital Social y las variables socioeconómicas del hogar. Este modelo se aleja de los propuestos por anteriores trabajos sobre pobreza y Capital Social Grooteart (1999) y Pritchett (1999), ya que la variable dependiente no es continua, como por ejemplo, el ingreso o el gasto del hogar. En cambio, este trabajo se acerca más a la literatura asociada a los factores determinantes de la pobreza (Cortés, 1997; Núñez y Espinosa, 2005), donde se busca estimar la probabilidad de que un hogar sea pobre, con base en un conjunto de variables socioeconómicas, como por ejemplo: el número y la edad de personas en el hogar (Núñez, Ramírez y Cuesta, 2005; Méndez y Jaramillo 2002; Carrizosa, 1987), la raza (Hall y Patrinos, 2005) o el lugar (Pérez, 2005).

Todo este tratamiento de la variable dependiente se hace con el objetivo de evitar la endogeneidad en el modelo y poder cuantificar con mayor exactitud y significancia el papel que desempeña el Capital Social.

\section{Modelos de elección discreta}

La modelización de variables cuyos valores son dicotómicos se denominan modelos de elección discreta. Según la función utilizada para la estimación de la probabilidad existe el modelo de probabilidad lineal truncado, el modelo Logit y el modelo Probit. En general se considera que detrás de la variable dependiente en estos modelos, $\mathrm{Y}$, se encuentra una variable no observable, I, que depende de un conjunto de variables explicativas $\mathrm{X} \_\mathrm{i}$ que toma ciertos valores si ha sobrepasado cierto umbral, como se expresa a continuación. 


$$
Y_{i}=\left\{\begin{array}{lll}
1 & \text { si } I_{i}^{*}>0 & \text { lo que ocurre cuando } X_{i} \beta+\varepsilon_{i}>0 \\
0 & \text { si } I_{i}^{*}<0 & \text { lo que ocurre cuando } X_{i} \beta+\varepsilon_{i}<0
\end{array}\right.
$$

Donde el supuesto sobre la distribución de $\varepsilon$, determina el tipo de modelo a estimar: si se supone una función de distribución uniforme, se utiliza el Modelo Lineal de Probabilidad truncado; si se distribuye como una normal con media cero y varianza uno, el modelo generado será un Probit; mientras que si se supone que se distribuye como una curva logística, se trataría de un modelo Logit. La hipótesis de que el umbral a superar por la variable latente sea cero, se puede modificar por cualquier otro valor sugiriéndose, en determinados estudios, que el valor crítico sea el definido por el término constante (Pérez, 2004).

Bajo el primer enfoque el modelo probabilístico quedaría definido (Medina, 2003)

$$
P_{i}=\operatorname{Pr} o b\left(Y_{i}=1\right)=\operatorname{Pr} o b\left(I_{i}^{*}>0\right)=\operatorname{Pr} o b\left(X_{i} \beta+\varepsilon_{i}>0\right)=F\left(X_{i} \beta\right)
$$

Con el modelo así definido, la variable endógena del modelo dicotómico representa la probabilidad de ocurrencia del fenómeno analizado .

\section{Estimación}

Para este trabajo, la función de estimación probabilística será

$$
Y=f(S, X) \quad Y=\alpha+\beta X_{i}+\theta S_{i}+\varepsilon
$$

Donde $\mathrm{Y}$ es una variable dicotómica, donde 1 es un hogar pobre y cero en caso contrario. Ésta es explicada por un vector factores del hogar X donde se encuentra el número y las características de las personas conformantes del hogar, la dotación de capital humano, el lugar donde se localiza el hogar, características del jefe de hogar etc. Asimismo S recoge la dotación de Capital Social del hogar, dada la participación del mismo en actividades comunitarias. Por su parte, $\beta$ y $\theta$ captan el aporte marginal de cada uno de estos factores a la probabilidad de ser pobre.

En función de lo anterior, se introdujeron los datos de las encuestas de hogares. A continuación se presentan los resultados obtenidos. 


\section{Resultados}

Con base en los ejercicios de estimación, los resultados de este trabajo incluyen las estadísticas sobre el comportamiento de la pobreza en Ecuador, así como de la participación social. Finalmente se presentan los resultados de las estimaciones de los modelos de elección discreta presentados anteriormente.

\section{Estadísticas generales de la pobreza}

En la siguiente gráfica se observa la evolución de la pobreza monetaria en Ecuador entre 2010 y 2012 calculada por el INE. Esta metodología define que la pobreza es la incapacidad de alcanzar cierto nivel de necesidades básicas (Banco Mundial, 1990) que se mide mediante el poder de adquirir una canasta de bienes y servicios básicos; cuantitativamente este límite se considera línea de pobreza (Alan, 1996)². En relación con la clasificación, los hogares que no pueden llegar a este límite se consideran pobres. Se aprecia que la reducción de la pobreza en Ecuador ha sido constante, desde el 32.8\% que se registraba en 2010 hasta el 25,3\% en junio de 2012.

1 Para consultar detalles sobre el cálculo de la pobreza monetaria en Ecuador, junto con el establecimiento de la línea de pobreza, se puede revisar en el siguiente link http://www.inec.gob.ec/ estadisticas/index.php?option $=$ com_remository Itemid $=$ yfunc $=$ downloadyid $=176 \mathrm{ychk}=2 \mathrm{f} 0 \mathrm{f} 6 \mathrm{e} 72 \mathrm{~d}$ 1dd5f1ba5e3acb573f5780byno_html=1ylang=es. En términos monetarios, la línea de pobreza en Ecuador se ubicó para el periodo de 2006 en los 54,64 dólares actualizable con el índice de precios al consumidor hasta el 2011. En 2012, esta línea se actualizó con base en la encuesta de calidad de vida hasta los $\$ 74.49$ dólares.

2 Al ingreso necesario para comprar una canasta de alimentos que cumplan con al menos 2141 calorías se le denomina línea de extrema. Esta se ubicó en los 45,15 dólares en el 2006 actualizable con el índice de precios al consumidor. 
Gráfica 2. Evolución y distribución del porcentaje de hogares que participan en actividades comunitarias. Porcentaje de Hogares en Pobreza Monetaria en Ecuador con datos sobre la línea de pobreza e inflación. Años 2010- Junio de 2012

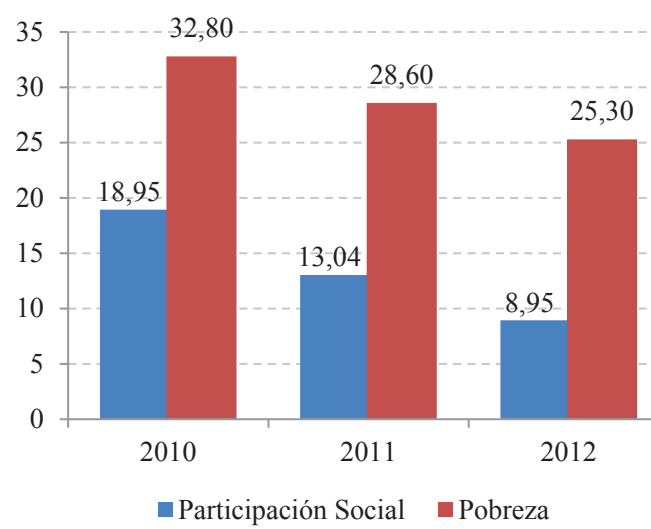

\begin{tabular}{|c|c|c|}
\hline Año & $\begin{array}{c}\text { Línea de } \\
\text { Pobreza } \\
\text { (USD) }\end{array}$ & $\begin{array}{c}\text { Inflación } \\
\text { Anual }\end{array}$ \\
\hline 2009 & 64.09 & $4.31 \%$ \\
\hline 2010 & 66.22 & $3.33 \%$ \\
\hline 2011 & 69.80 & $5.41 \%$ \\
\hline 2012 & 74.79 & $4.16 \%$ \\
\hline
\end{tabular}

Fuente: Banco Central de Ecuador. Instituto Nacional de Estadística con base en la Encuesta de Empleo, Subempleo y Desempleo 2010-2012.

\section{Estimaciones}

En la tabla 2 se muestran los resultados de los modelos implementados. La función de estimación se basa en modelos probabilísticos logit y probit con pruebas sobre 3’280,753 hogares en Ecuador en el periodo entre 2010 y 2012. En ésta, además se muestran las variables de control (independientes) y su efecto marginal sobre la probabilidad de ser pobre para los hogares en Ecuador entre el 2010 y el 2012, junto a su grado de significancia.

Entre los resultados sobre los que trata la hipótesis principal de este trabajo, no se encuentra evidencia estadística significativa que sustente que el Capital Social es un factor que contribuya a la reducción de la probabilidad de ser pobre. De hecho, el efecto encontrado es el contrario. Existe un 1\% adicional de que los hogares que pertenecen a organizaciones sociales sean pobres en contra de los que no están involucrados en dichas organizaciones. Esto no significa que las redes sociales que tejen estos hogares sean fútiles, como muestra el trabajo.

Un hecho que es relevante, es que año con año todos los hogares ecuatorianos, en promedio, han visto reducidas sus probabilidades de ser pobres desde el punto de vista monetario; es decir, los hogares han aumentado sus ingresos promedio de manera consistente, logrando alejarse. Por ejemplo en 2011 un hogar ecuatoriano 
tenía hasta un $4 \%$ menos de probabilidad en relación con el 2010, de ser pobre; este hecho es patente en el año 2012 donde la probabilidad es de -3,8\%

Otros factores deriesgo de que un hogar se considere pobre están:

- El lugar donde se encuentre el hogar. Solo por el hecho de estar ubicados en el área rural, los hogares tienen un 14,78\% más de ser pobres que los ubicados en el área urbana. Esto sucede a que los hogares localizados lejos de las cabeceras municipales no son capaces de disfrutar de la infraestructura que se aprovecha de las economías de escala, como las redes de agua, electricidad y alcantarillado (Grazzi, 2010).

- Asimismo, sucede con el género del jefe de hogar. Si es mujer, el hogar tienen una probabilidad mayor al 5,65\% de ser pobre. Esto se explica por las brechas salariales que se encuentran en el mercado laboral dada la discriminación salarial (Rivera, 2013; Galvis, 2010). Este comportamiento es propio de los jefes de hogar pertenecientes a pueblos indígenas, los cuales también cuentan con una alta probabilidad (entre un 13\%-14\%) de ser pobres, frente a otros grupos étnicos. Esto, a pesar de los programas sociales emprendidos por los gobiernos ecuatorianos para la atención de estos grupos (Ponce, y Bedi, 2010).

- El aumento de la edad del jefe de hogar puede contribuir al de la pobreza. Por cada año adicional el riesgo de ser pobre aumenta en un $0,34 \%$. Las mayores edades reducen la capacidad de ser empleado en el mercado laboral (Rivera, 2013), lo cual aumenta la exposición del hogar a la tenencia de menores recursos económicos.

- La mayor presencia de niños menores de 12 años en el hogar aumenta la probabilidad de ser pobre. Por cada $1 \%$ de participación adicional que tengan los niños en la composición del hogar, la probabilidad se incrementa hasta en un 69\%-67\%.

- La educación se muestra como uno de las mejores protecciones frente a la pobreza. Por ejemplo, una persona con grado educativo superior (incluye postgrados) tiene entre un $14 \%$ a un $19 \%$ menos de probabilidad de ser pobre, frente a un hogar cuyo jefe de hogar no cuente con ningún grado de educación. Este es un hecho común en la literatura internacional (García-Suaza, Guataquí, Guerra, y Maldonado, 2011) ya que los aumentos en el stock de capital humano de las personas les permite ser más productivos y rentabilizar así sus retornos en el mercado laboral. 
Tabla 2. Resultados de las Estimaciones

\begin{tabular}{|c|c|c|c|c|c|c|}
\hline \multirow[b]{2}{*}{ Variable } & \multirow[b]{2}{*}{ Características } & \multicolumn{2}{|c|}{ Logit } & \multicolumn{2}{|c|}{ Probit } & \multirow{2}{*}{$\begin{array}{c}\text { Variable } \\
\text { Base }\end{array}$} \\
\hline & & $\begin{array}{l}\text { Efecto } \\
\text { Marginal }\end{array}$ & $P>|z|$ & $\begin{array}{l}\text { Efecto } \\
\text { Marginal }\end{array}$ & $P>|z|$ & \\
\hline Capital Social & $\mathrm{Si}$ & $1.04 \%$ & 0.00 & $1.00 \%$ & 0.00 & No \\
\hline Área & Rural & $14.78 \%$ & 0.00 & $14.30 \%$ & 0.00 & Urbana \\
\hline Sexo & Mujer & $5.65 \%$ & 0.00 & $5.38 \%$ & 0.00 & Hombre \\
\hline Se Trasladó A Este Lugar & $\mathrm{Si}$ & $-4.80 \%$ & 0.00 & $-4.86 \%$ & 0.00 & No \\
\hline Recibe Dinero Del Exterior & $\mathrm{Si}$ & $-11.71 \%$ & 0.00 & $-11.43 \%$ & 0.00 & No \\
\hline \multicolumn{2}{|l|}{ Edad } & $0.34 \%$ & 0 & $0.37 \%$ & 0 & Continua \\
\hline \multicolumn{2}{|c|}{ Personas En La Vivienda } & $-2.33 \%$ & 0 & $-2.16 \%$ & 0 & Continua \\
\hline \multicolumn{2}{|c|}{ Proporción De Niños<12 } & $69.86 \%$ & 0 & $67.84 \%$ & 0 & Continua \\
\hline \multirow{2}{*}{ Año } & 2011 & $-3.94 \%$ & 0.00 & $-3.89 \%$ & 0.00 & 2010 \\
\hline & 2012 & $-3.80 \%$ & 0.00 & $-3.68 \%$ & 0.00 & \\
\hline \multirow{2}{*}{ Interacciones Capital y Año } & 2011 & $0.00 \%$ & 0.00 & $0.00 \%$ & 0.00 & 2010 \\
\hline & 2012 & $0.00 \%$ & 0.00 & $0.00 \%$ & 0.00 & \\
\hline \multirow{5}{*}{ Estado Civil } & Separado(a) & $-1.59 \%$ & 0.00 & $-1.31 \%$ & 0.00 & $\begin{array}{l}\text { Casado } \\
\text { (a) }\end{array}$ \\
\hline & Divorciado(a) & $-3.01 \%$ & 0.00 & $-3.14 \%$ & 0.00 & \\
\hline & Viudo(a) & $-5.90 \%$ & 0.00 & $-5.66 \%$ & 0.00 & \\
\hline & Unión Libre & $-0.34 \%$ & 0.00 & $-0.35 \%$ & 0.00 & \\
\hline & Soltero(a) & $4.84 \%$ & 0.00 & $4.72 \%$ & 0.00 & \\
\hline \multirow{8}{*}{ Educación } & $\begin{array}{c}\text { Centro De } \\
\text { Alfabetización }\end{array}$ & $2.55 \%$ & 0.00 & $2.41 \%$ & 0.00 & Ninguno \\
\hline & Primaria & $-5.77 \%$ & 0.00 & $-5.99 \%$ & 0.00 & \\
\hline & Educación Básica & $-3.91 \%$ & 0.00 & $-4.05 \%$ & 0.00 & \\
\hline & Secundaria & $-11.66 \%$ & 0.00 & $-12.02 \%$ & 0.00 & \\
\hline & Educación Media & $-4.16 \%$ & 0.00 & $-4.87 \%$ & 0.00 & \\
\hline & $\begin{array}{l}\text { Superior No } \\
\text { Universitaria }\end{array}$ & $-18.22 \%$ & 0.00 & $-19.13 \%$ & 0.00 & \\
\hline & $\begin{array}{c}\text { Superior } \\
\text { Universitaria }\end{array}$ & $-14.59 \%$ & 0.00 & $-14.54 \%$ & 0.00 & \\
\hline & Post-grado & $-14.40 \%$ & 0.00 & $-14.19 \%$ & 0.00 & \\
\hline \multirow{7}{*}{ Etnia } & Afro ecuatoriano & $-10.48 \%$ & 0.00 & $-11.05 \%$ & 0.00 & Indígena \\
\hline & Negro & $-13.65 \%$ & 0.00 & $-14.32 \%$ & 0.00 & \\
\hline & Mulato & $-14.07 \%$ & 0.00 & $-14.76 \%$ & 0.00 & \\
\hline & Montubio & $-14.42 \%$ & 0.00 & $-15.01 \%$ & 0.00 & \\
\hline & Mestizo & $-18.54 \%$ & 0.00 & $-18.33 \%$ & 0.00 & \\
\hline & Blanco & $-7.92 \%$ & 0.00 & $-8.17 \%$ & 0.00 & \\
\hline & Otro & $-15.42 \%$ & 0.00 & $-14.53 \%$ & 0.00 & \\
\hline \multirow{5}{*}{ Sector } & Sector Formal & $-41.01 \%$ & 0.00 & $-41.58 \%$ & 0.00 & \multirow{5}{*}{$\begin{array}{c}\text { Inactivos } \\
\text { o } \\
\text { menores } \\
\text { de } 10 \\
\text { años }\end{array}$} \\
\hline & Sector Informal & $-28.40 \%$ & 0.00 & $-28.75 \%$ & 0.00 & \\
\hline & $\begin{array}{c}\text { Servicio } \\
\text { Doméstico }\end{array}$ & $-23.85 \%$ & 0.00 & $-25.63 \%$ & 0.00 & \\
\hline & Desempleado & $4.83 \%$ & 0.00 & $4.68 \%$ & 0.00 & \\
\hline & $\begin{array}{l}\text { Ocupado No } \\
\text { Clasificado }\end{array}$ & $-22.17 \%$ & 0.00 & $-23.54 \%$ & 0.00 & \\
\hline \multirow{4}{*}{ Indicadores De La Estimación } & \multicolumn{2}{|c|}{ Individuos evaluados } & \multirow{2}{*}{\multicolumn{2}{|c|}{$3,280,753$}} & $3,280,753$ & \\
\hline & \multicolumn{2}{|c|}{ Wald Chi2(54) } & & $2,132,621$ & $2,434,453$ & \\
\hline & \multicolumn{2}{|c|}{ Máximum Likelihood R2 } & \multicolumn{2}{|c|}{0.25} & 0.249 & \\
\hline & \multicolumn{2}{|c|}{ \% De Correcta Clasificación } & \multicolumn{2}{|c|}{$75.27 \%$} & $75.23 \%$ & \\
\hline
\end{tabular}

Fuente: Cálculos del Autor con base en las encuestas de empleo de Ecuador.

Para

citar este

artículo:
Botello Peñaloza, Héctor Alberto (2013). Capital Social y Pobreza en Ecuador, 2010-2012.

Ánfora 20(35), 93-115. Universidad Autónoma de Manizales. ISSN 0121-6538. 
- Los hogares, cuyo jefe de hogar trabaja en el sector formal de la economía, tienen una probabilidad de menos de un $41,6 \%$ de ser pobre frente a un hogar cuyo jefe se encuentre inactivo, seguidos por los informales con el 28,4\%. Mientras, los que están desempleados incrementan un 4,83\% su probabilidad. Este hallazgo resalta la importancia de la participación laboral de los hogares para salir de la pobreza.

\section{Conclusiones}

Sobre la tesis central de este trabajo, el capital social de los hogares Ecuatorianos no resultó ser un componente significativo en la reducción de la pobreza. No obstante, sí se evidenció una reducción continúa de la probabilidad de pobreza de los hogares ecuatorianos estudiados, cercano al 4\% anual. Esta estimación es neta ya que se ha obtenido controlando diferentes factores socioeconómicos y características de los hogares.

Se puede decir que los hogares más propensos a participar en actividades comunitarias están caracterizados con menores ingresos. Sus jefes de hogar son hombres, de etnia indígena y con bajos grados educativos. La educación y la composición del hogar pueden ser factores significativos que afectan la probabilidad de ser pobre de los hogares. Además, etnias como la indígena presentan vulnerabilidades significativamente más altas frente a otros grupos, mientras que la tipología del trabajo formal es otro de las mejores protecciones a la pobreza.

De los resultados obtenidos, se pueden establecer las siguientes líneas y recomendaciones para trabajos posteriores:

- La primera recomendación es la definición y la cuantificación del Capital Social. Dadas las múltiples interpretaciones del concepto (Woolcock y Narayan, 2000) se tendrían que relacionar diferentes indicadores basados en los conceptos, para vincularlo con la pobreza.

- La restricción de lo anterior es la falta de información correspondiente a las relaciones sociales de los individuos y hogares, ya que estas son dinámicas y dispersas. Por esta razón, es de esperar que esta problemática se convierta en una constante en estas investigaciones.

- Es necesario continuar el debate sobre la medición de la pobreza más allá de los ingresos (Sen, 1999). Sobre el particular, los índices multidimensionales evalúan una mayor cantidad de necesidades de los individuos (Alkire y Foster, 2011; Bourguignon y Chakravarty, 2003; Duclos, Sahn y 
Younger, 2006; Tsui, 2002). Esta mejor definición, favorecería el establecimiento de vínculos más robustos entre el Capital Social y la pobreza, lo que puede estar perjudicando los resultados de esta investigación .

- Establecer la intensidad con la cual se da el Capital Social en las comunidades. La cuantificación de este aspecto se podría estimar con el número de horas que dedican los hogares a las asociaciones comunitarias. Ya que no son iguales las relaciones sociales que un hogar que dedica 20 horas a la semana a afianzar sus lazos, que uno que sólo dedica una hora.

\section{Referencias}

Alan, G. (1996). The origin of the poverty line. Economic History Review, (49), 726.

Alkire, S., y Foster, J. (2011). Counting and multidimensional poverty measurement. Journal of Public Economics 95(7), 476-487.

Arriagada, I. (2005). Aprender de la experiencia: el capital social en la superación de la pobreza. CEPAL.

Banco Central de Ecuador (BCE) (junio, 2012) Boletín Anuario N 34. Edición Electrónica Recuperado de: http://www.bce.fin.ec/frame.php?CNT=ARB0000006.

Banco Mundial (1990). Informe sobre el desarrollo mundial 1990. La pobreza; Report on world development 1990. Poverty (13). Banco Mundial.

Bourguignon, F., y Chakravarty, S. R. (2003). The measurement of multidimensional poverty. The Journal of Economic Inequality 1(1), 25-49.

Carrizosa, M. (1987). Evolución y Determinantes de la Pobreza en Colombia. El Problema Laboral Colombiano. Bogotá: SENA, DNP, Contraloría General de la República.

Caughy, M. O., y O'Campo, P. J. (2006). Neighborhood poverty, social capital, and the cognitive development of African American preschoolers. American Journal of Community Psychology 37(1-2), 141-154. doi:10.1007/s10464-005-9001-8

Conferencia Económica para América Latina, CEPAL (2010). Panorama Social de América Latina.

Chantarat, S., y Barrett, C. B. (2012). Social network capital, economic mobility and poverty traps. Journal of Economic Inequality, 10(3), 299-342. doi:10.1007/s10888-011-9164-5

Cleaver, F. (2005). The inequality of social capital and the reproduction of chronic poverty. World Development, 33(6), 893-906.

Coleman, J. S. (1988). Social capital in the creation of human capital. American journal of sociology, S95-S120. 
Collier, P. (2002). Social capital and poverty: a microeconomic perspective. En The role of social capital in development. An empirical assessment, (19-41).

Cortés, F. (1997). Determinantes de la pobreza de los hogares. México, 1992. Revista mexicana de sociología, 131-160.

Das, R. J. (2004). Social capital and poverty of the wage-labour class: problems with the social capital theory. Transactions of the Institute of British Geographers, 29(1), 27-45. doi:10.1111/j.0020-2754.2004.00112.x

Dasgupta, P. (2000). Economic progress and the idea of social capital. Social capital. A multifaceted perspective, 325-424.

Duclos, J.-Y., Sahn, D. E., y Younger, S. D. (2006). Robust multidimensional poverty comparisons. The economic journal, 116(514), 943-968.

Galvis, L. A. (2010). Diferencias salariales por género y región en Colombia: una aproximación con regresión por cuantiles (Wage Gap by Gender and Region in Colombia: A Quantile Regression Approach). Available at SSRN 1860356.

García-Suaza, A. F., Guataquí, J. C., Guerra, J. A., y Maldonado, D. (2011). Beyond the Mincer equation: the internal rate of return to higher education in Colombia. Education Economics, (ahead-of-print), 1-17.

Granovetter, M. S. (1973). The strength of weak ties. American journal of sociology, 13601380.

Granovetter, M. S. (1985). Economic action and social structure: the problem of embeddedness. American journal of sociology, 481-510.

Granovetter, M. S. (1995). The Economic Sociology of Firms and Entrepreneurs. En A. Portes. The Economic Sociology of Immigration (pp. 128 - 65). New York: Russell Sage Foundation

Grazzi, M. (2010). Infraestructura, crecimiento económico y pobreza: ¿ Por qué es clave invertir en infraestructura en Venequela? (No. 79103). Inter-American Development Bank.

Gil-Lacruz, M., y Gil-Lacruz, A. I. (2006). Capital humano y capital social, implicaciones en el crecimiento económico. Revista del Ministerio de Trabajo y Asuntos Sociales, (61), 93-104.

Grooteart, C., (1999), Social Capital, Household Welfare and Poverty in Indonesia. World Bank Social Capital Initiative. Discussion Paper.

Grooteart, C. (2001). The missing link? Social capital and participation in everyday life, 8.

Grooteart, C., y Narayan, D., (2004). Local Institutions, Poverty and Household Welfare in Bolivia. World Development: 32(7), 1179-1198. 
Hall, G., y Patrinos, H. A. (2005). Pueblos indígenas, pobreza y desarrollo bumano en América Latina: 1994-2004. Ed: Banco Mundial.

Hassan, R., y Birungi, P. (2011). Social capital and poverty in Uganda. Development Southern Africa, 28(1), 19-37. doi:10.1080/0376835X.2011.545168.

Knack, S. (2002). Social capital, growth and poverty: A survey of cross-country evidence. The role of social capital in development: An empirical assessment, 42-82.

Knack y Keefer (1997). Does Social Capital Have an Economic Payoff? A Cross-Country Investigation. Quarterly Journal of Economics, (112), 1251-88.

Lahn, J. (2012). Poverty, Work and Social Networks: The Role of Social Capital for Aboriginal People in Urban Australian Locales. Urban Policy and Research, 30(3), 293-308. doi:10.1080/08111146.2012.673483

Londoño J. L. (1997) Violencia, Psychis y Capital social. Segunda conferencia Anual de desarrollo Económico. Banco Mundial. Washington

Medina, E. (2003). Modelos de elección discreta. Publicaciones Económicas, 26. Madrid: Universidad Autónoma de Madrid.

Méndez, A. N., y Jaramillo, C. R. (2002). Determinantes de la pobreza en Colombia: años recientes. United Nations Publications.

Mideros, M. (2012). Ecuador: Definición y medición multidimensional de la pobreza, 2006-2010. Revista CEPAL (108). Comisión Económica para América Latina y el Caribe (CEPAL)

Narayan, D. (1997). Voices of the Poor: Poverty and Social Capital in Tanzania. En Banco Mundial. Desarrollo Ambientalmente Sostenible. Monografía No. 20. Washington, D.C.: Banco Mundial.

Narayan, D., y Pritchett, L. (1999). Cents and sociability: Household income and social capital in rural Tanzania. Economic development and cultural change, 47(4), 871-897.

Núñez, J., Ramírez, J. C., y Cuesta, L. (2005). Determinantes de la pobreza en Colombia, 1996-2004. Documento CEDE, 60.

Núñez, J., y Espinosa, S. (2005). Determinantes de la pobreza y la vulnerabilidad. Misión para el diseño de una estrategia para la reducción de la pobreza y la desigualdad. Departamento Nacional de Planeación.

Pérez, G. J. (2005). Dimensión espacial de la pobreza en Colombia. Documentos de trabajo sobre economía regional, (54).

Pérez, C. (2004). Técnicas de análisis multivariante de datos. Aplicaciones con SPSS. Madrid: Universidad Complutense de Madrid.

Ponce, J., y Bedi, A. S. (2010). The impact of a cash transfer program on cognitive achievement: The Bono de Desarrollo Humano of Ecuador.Economics of Education. Review, 29 (1), 116-125. 
Putnam, R. D. (1993). Making Democracy Work: Civic Traditions in Modern Italy. Princeton: Princeton University Press.

Putnam, R. D. (2001). Social Capital: Measurement and Consequences. Canadian Journal of Policy Research. Spring

Restrepo, P. R. (1998). Capital social, crecimiento económico y políticas públicas. Lecturas de economía, (48), 33-66.

Rivera, J. (2013). Teoría y práctica de la discriminación en el mercado laboral ecuatoriano (2007-2012). Analitika, 5(1), 3-18.

Rodrik, D. (1997). Trade, social insurance, and the limits to globalization (No. w5905). National Bureau of Economic Research.

Romer, P. M. (1990). Endogenous technological change. Journal of political Economy, S71S102.

Saracostti, M. (2010). Social capital as a strategy to overcome poverty in Latin America - An overview. International Social Work, 50(4), 515. doi:10.1177/0020872807077911

Schneider, J. A. (2005). Getting beyond the training vs. work experience debate: The role of labor markets, social capital, cultural capital, and community resources in long-term poverty. Journal of Women Politics y Policy, 27(3-4), 41-53. doi:10.1300/ J501v27n03_04

Sen, A. (1999). Development as freedom. Oxford: University Press

Solow, R. M. (1957). Technical change and the aggregate production function. The review of Economics and Statistics, 39(3), 312-320.

Solow, R. M. (2000). Notes on social capital and economic performance. Social capital: A multifaceted perspective, 6(10). Recuperado de http://books.google.es/books?hl=e nylr=yid=6PZ8bvQQmxECyoi=fndypg=PA6ydq=related:EYQ5M5WU5Rw:sch olar.google.com/yots=EGpeHhiKSnysig=hqg571fGExgeM0mBAZPSEJfIGpE

Tsui, K. (2002). Multidimensional poverty indices. Social Choice and Welfare, 19(1), 69-93.

Woolcock, M., y Narayan, D. (2000). Social capital: Implications for development theory, research, and policy. The world bank research observer, 15(2), 225.

Para $\mid$ Botello Peñaloza, Héctor Alberto (2013). Capital Social y Pobreza en Ecuador, 2010-2012. 